(Raynaud's phenomenon, pulmonary interstitial involvement, digital ulcers, diges tive alterations, presence of sclerodermic renal crisis) and activity index variables (modified Rodnan score, HAQ-DI, SHAQ-VAS)

Results: Four patients were included ( $75 \%$ women). The median age at the time of the AHSCT was 36.5 years (range $27-51$ ). In all cases, the initial diagnosis was diffuse cutaneous ES, refractory to corticosteroids and at least one DMARD. Prior to autologous hematopoietic stem-cells transplantation, the clinical manifestations presented were a) severe Raynaud's phenomenon (100\%) with significant joint and cutaneous involvement; b) digital ulcers (50\%); c) interstitial lung disease $(50 \%)$ and d) sclerodermal renal crisis $(25 \%)$. In 3 of the cases $(75 \%)$ the antitopoisomerase antibodies were positive. The conditioning treatment for the autologous hematopoietic stem-cells transplantation was cyclophosphamide at high doses $(50 \mathrm{mg} / \mathrm{kg} \times 4$ days) and anti-thymocyte globulin. In 3 patients $(75 \%)$ there were slight post-transplant complications (febrile neutropenia, diarrhoea) after a median follow-up of 6.5 years (range $1-15$ ).

The response to AHSCT is summarised in table 1. All patients showed values $<1$ in the Health Assessment Questionnaire on the Disability Index (HAQ-DI), in 75\% with a modified Rodnan skin score (mRSS) lower than 7.

Abstract AB0718 - Table 1

\begin{tabular}{lccccc}
\hline PATIENT & $\begin{array}{c}\text { FOLLOW- } \\
\text { UP }\end{array}$ & $\begin{array}{c}\text { MODIFIED } \\
\text { RODNAN } \\
\text { SKIN SCORE } \\
\text { PRE- } \\
\text { TRASPLANT }\end{array}$ & $\begin{array}{c}\text { MODIFIED } \\
\text { RODNAN SKIN } \\
\text { SCORE POST } \\
\text { TRASPLANT }\end{array}$ & $\begin{array}{c}\text { HAQ-DI } \\
\text { POST } \\
\text { TRASPLANT }\end{array}$ & $\begin{array}{c}\text { SHAQ-VAS } \\
\text { POST } \\
\text { TRASPLANT }\end{array}$ \\
\hline Patient 1 & 15 years & Unknown & $1 / 51$ & 0,25 & $4 / 13$ \\
Patient 2 & 8 years & Unknown & $7 / 51$ & 0 & $3 / 13$ \\
Patient 3 & 5 years & $28 / 51$ & $6 / 51$ & 0 & $0 / 13$ \\
Patient 4 & 1 year & $33 / 51$ & $20 / 51$ & 0,50 & $7 / 13$ \\
\hline
\end{tabular}

Conclusions: Autologous hematopoietic stem-cells transplantation can be a therapeutic option in refractory and severe SS. These hopeful data must be ratified in larger studies.

Disclosure of Interest: None declared

DOI: 10.1136/annrheumdis-2018-eular.7007

\section{AB0719 CLOSE TEMPORAL ASSOCIATION BETWEEN SILICONE COSMETIC SURGERY AND SYSTEMIC SCLEROSIS ONSET}

A. Sulli, S. Paolino, E. Alessandri, B. Ruaro, M. Pendolino, M. Cutolo, C. Pizzorni. Research Laboratory and Academic Division of Clinical Rheumatology, Department of Internal Medicine, University of Genova, San Martino Polyclinic Hospital, Genova, Italy

Background: The pathogenesis of systemic sclerosis (SSc) still remains unclear, however It is increasingly thought to result from interactions between environmental factors and epigenetic features leading to the onset and progression of SSc in genetically susceptible patients. ${ }^{1}$ Case reports of women with silicone breast implants who developed SSc have been published, but several case-control series and prospective studies in connective tissue diseases (including SSc) failed to find an increased risk of SSc associated with silicone cosmetic surgery. ${ }^{2}$ However, several byas may be recognised in these studies, i.e. heterogeneous cohorts of enrolled patients not selective for SSc, non homogeneous either disease duration or disease stage at study entry. For these reason the possible effect of silicone implants as immune adjuvants is not clear. ${ }^{3}$

Objectives: Retrospective study to find out patients who developed SSc after cosmetic surgery.

Methods: The clinical files of 110 female patients with systemic sclerosis were retrospectively evaluated. Among these, four patients showing a history of silicone cosmetic surgery (3.6\%) were identified, and clinical data collected.

Results: The clinical data of the four patients are below reported. 1. LS 28 year old female who underwent cosmetic breast prosthesis: two years later she complained of Raynaud' phenomenon (RP), and one more year later aggressive diffuse cutaneous SSc, along with anticentromere antibodies (ACA) positivity. 2. PJ 38 year old female who underwent cosmetic breast prosthesis: one year later she experienced RP and one more year later aggressive diffuse cutaneous SSc; antinuclear antibodies were positive with a speckled patter, but specific SSc-related autoantibodies negative. 3 . BS 33 year old female who underwent cosmetic breast prosthesis: two years later she complained of RP and one more year later limited cutaneous SSc with ACA positivity; SSc clinical condition partially improved and its progression stopped after breast prosthesis removal. 4. CM 58 year old female who underwent cosmetic lip silicone application: one year later she complained simultaneous onset of RP and aggressive diffuse cutaneous SSc with anti-Topoisomerase positivity; she died during follow-up.

Conclusions: This study reports a prevalence of $3.6 \%$ of silicone cosmetic surgery before SSc onset. The close temporal association between silicone implant and disease development suggests a possible role of silicone in SSc pathogenesis. Specifically addressed clinical studies or big-data studies need to rule out this matter.

REFERENCES:

[1] Denton C, et al. Lancet 2017; 390: 1685-99.

[2] Marie I, et al. Semin Immunopathol 2015; 37:463-473.

[3] Watad A, et al. Lupus. 2017; 26:675-681.

Disclosure of Interest: None declared

DOI: 10.1136/annrheumdis-2018-eular.7433

\section{AB0720 SYSTEMIC SCLEROSIS AND CANCER DEVELOPMENT. A SINGLE-CENTRE EXPERIENCE}

E. Pelechas, E. Kaltsonoudis, P.V. Voulgari, A.A. Drosos. Rheumatology Clinic, Department Of Internal Medicine, MEDICAL SCHOOL, UNIVERSITY OF IOANNINA, Ioannina, Greece

Background: Systemic Sclerosis (SSc) is an autoimmune connective tissue disease with multisystem involvement, and sometimes devastating results. In bibliography there are reports that scleroderma patients present a higher incidence of risk for cancer when compared with the general population. However, different estimates have been reported.

Objectives: The purpose of the present study was to evaluate the frequency of cancer development (CD) in a cohort of patients with SSc.

Methods: Patients that fulfilled the 2013 American College of Rheumatology/ EUropean League Against Rheumatism criteria for SSc and were followed up since 1999, were included. Date of disease onset, disease duration, autoantibodies, age, pulmonary hypertension, comorbidities and the type of CD have all been taken into account, during the period 1991-2016.

Results: Seventy-nine SSc patients have been included. 46 with limited (IcSSc) and 33 with diffuse cutaneous Systemic Sclerosis (dcSSc). Six of them, (7.6\%) developed different types of cancer. Most of them were adenocarcinomata. More specifically, 2 developed pulmonary adenocarcinoma (1 with IcSSc and 1 with dcSSc), 1 follicular carcinoma of the thyroid gland (IcSSc), 1 colorectal adenocarcinoma (IcSSc), 1 B-cell lymphoma (MALT Iymphoma), and 1 prostate adenocarcinoma (dcSSc). Five out of six were female patients. Mean age at the time of cancer diagnosis was 66.8-years-old, while SSc has been diagnosed at the mean age of 49.4 years. Mean time of developing any type of cancer was 15.8 years after SSc diagnosis. The diagnosis of cancer was done the last 20 months. All patients were non-smokers, had gastro-oesophageal reflux disease and pulmonary fibrosis, while $4 / 6$ had also pulmonary hypertension and were under treatment with phosphodiesterase 5 inhibitors and bosentan. Scleroderma patients with $C D$ have been referred to the corresponding oncology clinic for further treatment.

Conclusions: The present study on SSc and CDE provides data showing a potential association between the two entities. We found a high frequency of can cer development in patients with SSc (7.6\%). Thus, a careful monitoring and screening is required when physicians follow-up scleroderma patients.

Disclosure of Interest: None declared

DOI: 10.1136/annrheumdis-2018-eular.3396

\section{AB0721 TRANSFER OF SYSTEMIC SCLEROSIS AFTER ALLOGENEIC BONE MARROW TRANSPLANTATION}

A. Silva Riveiro, J.C. Nieto González, J. Martínez Barrio, A. López-Cerón Cofiño, L. A. Torrens Cid, C. Soleto Kharkovskaya, B. Serrano Benavente, R.D. González Benítez, C.M. González Fernández, F.J. López-Longo, I. Monteagudo Sáez. Rheumatology, HOSPITAL GENERAL UNIVERSITARIO GREGORIO MARAÑON, MADRID, Spain

Background: It is accepted that donor-derived immunity is transferred with allogeneic bone marrow transplantation (BMT) ${ }^{1}$

Objectives: To show evidence of the transfer of systemic sclerosis by allogeneic BMT

Methods: In this report we describe a pacient with T acute lymphoblastic leukaemia who underwent BMT and developed systemic sclerosis.

Results: 34-year-old man in complete remission from a T acute lymphoblastic leukaemia treated with allogeneic BMT from his mother in february of 2012.

First seen in november 2017 for digital ulcers that appeared one year before. He presented two necrotic ulcers: one on the second finger of the left hand and other on the third finger of the right hand (IMAGE 1). He was admited to receive intravenous prostaglandins and complete the study.

After the BMT he developed Raynaud's phenomenon and in the examination he only presented facial and corporal telangiectasia, attributed before to chronic graft versus host disease (cGVHD). 
The analysis showed ANA 1/640 centromere pattern, anticentromere antibodies, reumatoid factor (RF) $(652 \mathrm{UI} / \mathrm{mL})$ and $\mathrm{C} 3$ of $86.4 \mathrm{mg} / \mathrm{dL}$. Previously to the BMT he had negative ANA, but we do not know the rest of the previous autoimmunity. On the videocapillaroscopy we observe an active scleroderma pattern (IMAGE 23)

He was diagnosed with systemic sclerosis based on Raynaud's phenomenon, digital ulcers, anticentromere antibodies and abnormal nailfold capillaries. He had not familiar background of connective tissue diseases, but his mother presented Raynaud's phenomenon since she was thirty. So we studied her.

She presented facial telangiectasia, puffy fingers and fingertip pitting scars and the same autoantibodies: ANA 1/320 centromere pattern, anticentromere antibodies, RF (159 Ul/mL) and consumption of C3 (74.3 mg/dL) and C4 (7.6). We do not have previous autoimmune studies of her. On the videocapillaroscopy we observed a late scleroderma pattern (IMAGE 4-5).

She was also diagnosed with systemic sclerosis, based on Raynaud's phemomenon, puffy fingers, fingertip pitting scars and facial telangiectasia, anticentromere antibodies and abnormal naifold capillaries.

Conclusions: Experimental animal studies and human clinical reports have described the transfer of immune-mediated diseases from affected donors to unaffected recipients, because of that the importance of screening this diseases in the donor before a $\mathrm{BMT}^{2}$

To our knowledge, this will be the first described case of transmission of systemic sclerosis by this mechanism. However, other explanations should also be taken into consideration. This include recipient's own persistent intrathymic lymphocyte population that may produce autoantibodies and autoimmunity in the context of cGVHD. However, this last hypothesis is not supported since there is a familiar background and presence of anticentromere antibodies.

\section{REFERENCES :}

[1] Sherer, Y. and Shoenfeld, Y. (1998). Autoimmune diseases and autoimmunity post-bone marrow transplantation. Bone Marrow Transplantation.

[2] Hough, R.(2005). Haemopoietic stem cell transplantation in autoimmune diseases: a European perspective. British Journal of Haematology.

Disclosure of Interest: None declared

DOI: 10.1136/annrheumdis-2018-eular.7042

\section{AB0722 CAPILLAROSCOPY AND PULMONARY ARTERIAL HYPERTENSION IN SYSTEMIC SCLEROSIS: A SYSTEMATIC REVIEW}

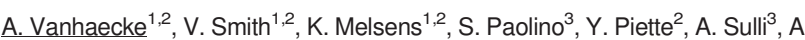
C. Trombetta ${ }^{3}$, F. De Keyser ${ }^{1,2}$, E. Vandecasteele ${ }^{4}$, M. Cutolo ${ }^{3}$, on behalf of the EULAR study group on microcirculation in Rheumatic diseases. ${ }^{1}$ Department of Internal Medicine, Ghent University; ${ }^{2}$ Department of Rheumatology, Ghent University Hospital, Ghent, Belgium; ${ }^{3}$ Research Laboratory And Academic Division Of Clinical Rheumatology, Department Of Internal Medicine, Irccs San Martino Aou, University Of Genoa, Genoa, Italy, ${ }^{4}$ Department of Cardiology, Ghent University Hospital, Ghent, Belgium

Background: At this very moment, no systematic review evaluating the role of nailfold videocapillaroscopy (NVC), with standardised definitions, in pulmonary arterial hypertension (PAH) has been published.

Objectives: To systematically identify and review all available literature evaluating the role of capillaroscopy in PAH in SSc, according to the definitions of the EULAR study group on microcirculation in Rheumatic diseases.

Methods: A systematic literature search was performed in Pubmed, EMBASE and Web of Science. All retrieved articles were screened on title, abstract and fulltext level. Reference lists and google scholar were additionally searched. Original research papers that documented an association between NVC and PAH (confirmed on right heart catheterization [RHC]) in SSc were included. Subsequently, NVC parameters were subdivided in quantitative (density, dimension, morphology and haemorrhages), semi-quantitative (NVC score) and qualitative assessment (presence of scleroderma pattern, severity of scleroderma pattern and worsening of scleroderma pattern) according to the definitions of the EULAR study group on microcirculation in Rheumatic diseases.

Results: The systematic search identified 215 unique search results, of which 171 references were withheld after title screening. Abstract screening resulted in 51 references, only 19 were eligible for full-text review. Finally, 9 references were included in the final analysis after full-text screening $(n=7)$ and bibliographic and google scholar search $(n=2)$ (see table 1 ).

Regarding cross-sectional studies, density has been evaluated in 5 studies, with no unequivocal results for mean density; avascular score has been unequivocally associated with PAH. Dimension has been evaluated in 4 studies, with no unequivocal results. Morphology has been evaluated in 1 study and has been unequivocally associated with $\mathrm{PAH}$. Haemorrhages and scleroderma pattern has been evaluated in 1 and 2 studies respectively, with no association. Severity of scleroderma pattern has been evaluated in 3 studies and has been unequivocally associated with $\mathrm{PAH}$

Regarding longitudinal studies, density (i.e. capillary loss) has been evaluated in 2 studies and has been unequivocally associated with incident PAH. Dimension and haemorrhages have been evaluated in 1 study both, with no association. Mor phology has been evaluated in 2 studies, with no unequivocal results. Worsening of scleroderma pattern has been evaluated in 2 studies and has been unequivocally associated with incident $\mathrm{PAH}$.

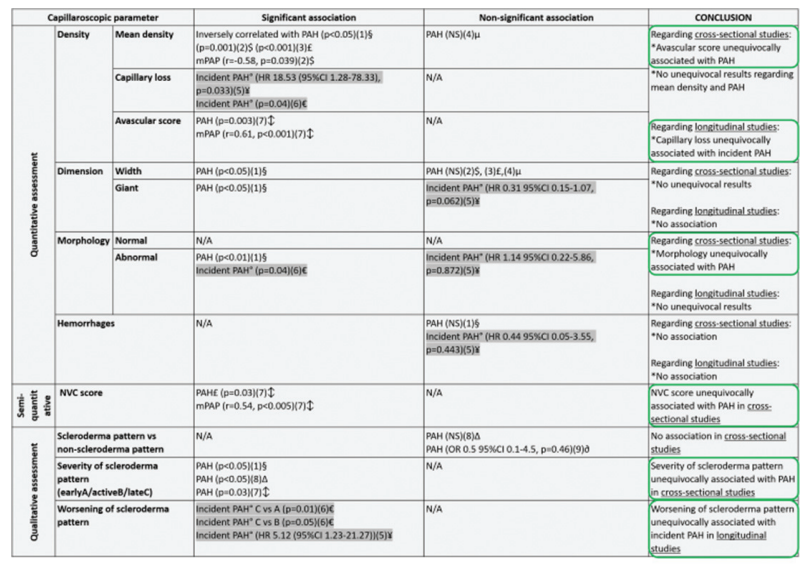

Abstract AB0722 - Figure

Conclusions: This systematic literature review, on behalf of the EULAR study group on microcirculation in Rheumatic diseases, is the first to investigate unequivocal associations between (incident) PAH and capillaroscopic alterations in a standardised way. Unequivocal associations were found in cross-sectional studies between avascular score, morphology, NVC score and severity of scleroderma pattern and PAH and in longitudinal studies between capillary loss and worsening of scleroderma pattern and incident $\mathrm{PAH}$

Disclosure of Interest: None declared

DOI: 10.1136/annrheumdis-2018-eular.6210

\section{$\mathrm{AB} 0723$ \\ SEROPREVALENCE OF EPSTEIN-BARR VIRUS AND CYTOMEGALOVIRUS IN SYSTEMIC SCLEROSIS PATIENTS: PRELIMINARY RESULTS}

A.C. Trombetta ${ }^{1}$, V. Tomatis ${ }^{1}$, E. Alessandri ${ }^{1}$, S. Paolino ${ }^{1}$, C. Pizzorni ${ }^{1}$, M. Ghio ${ }^{1}$, B. Ruaro ${ }^{1}$, M. Patane ${ }^{1}$, E. Gotelli ${ }^{1}$, F. Goegan ${ }^{1}$, A. Sulli ${ }^{1}$, V. Smith ${ }^{2}$, M. Cutolo ${ }^{1}$. ${ }^{1}$ Research Laboratory and Academic Division of Clinical Rheumatology, Department of Internal Medicine, IRCCS Polyclinic Hospital San Martino, University of Genoa, Genoa, Italy; ${ }^{2}$ Department of Rheumatology, University Hospital of Ghent, Ghent, Belgium

Background: Epstein-Barr Virus (EBV) and cytomegalovirus (CMV) are among the most diffused virus in humans, with prevalence of $90 \%$ and $80 \%$ respectively in adult immunocompetent population ${ }^{1,2}$. Hypothesis for a role of EBV and CMV in systemic sclerosis pathogenesis was reported ${ }^{3}$.

Objectives: To evaluate the prevalence of EBV and CMV in a population of patients affected by SSc. As an exploratory aim, correlations with available clinical variables were evaluated.

Methods: A total of 49 patients (44 females and 5 males, age 65,6 \pm 9 years, 34 with limited cutaneous involvement and 14 with diffused cutaneous involvement) diagnosed according to the 2013 ACR/EULAR criteria ${ }^{4}$ were randomly enrolled at their follow-up visit for a further more detailed analysis. After consent, all participants undergone serological testing for VCA-IgM, VCA-IgG and EBNA-IgG and CMV IgM and IgG, to determine prior or actual EBV and/or CMV infection. All SSc patients undergone also to clinical examination and instrumental evaluations according to SSc clinical standard follow up.

Results: Results for EBV seroprevalence were: VCA-IgM negative $=43(87 \%)$ positive $=4(8,2 \%)$, borderline $=2(4,1 \%)$ in all SSc patients. VCA-IgG resulted positive in 48 patients $(98 \%)$ and EBNA-IgG resulted positive in $40(81,6 \%)$ patients, borderline in $4(8,2 \%)$ and negative in $5(10,2 \%)$ patients. Forty-six $(93,9 \%)$ patients resulted positive for CMV-lgG and 22 (44,9\%) showed detectable CMV IgM. Consequently, EBV and CMV seroprevalence was significantly higher $(\mathrm{p}=0.01$ and $\mathrm{p}=0.032$ respectively) than that calculated in general population ( $90 \%$ and $80 \%$ respectively). Interestingly, the presence of higher CMV-IgM corre lated linearly with higher pulmonary arterial pressure values $(p=0.036)$ and was different in patients with or without sicca syndrome $(p=0.012)$ and arthralgias 